\title{
Relationship between crack propagation and the stress intensity factor in cutting parallel to the grain of hinoki (Chamaecyparis obtusa)
}

\author{
Masumi Minagawa $^{1,2} \cdot$ Yosuke Matsuda $^{3} \cdot$ Yuko Fujiwara $^{1} \cdot$ Yoshihisa Fujii $^{1}$
}

Received: 23 December 2017 / Accepted: 22 August 2018 / Published online: 25 September 2018

(c) The Japan Wood Research Society 2018

\begin{abstract}
The method of digital image correlation (DIC) was applied to the digital image of orthogonal cutting parallel to the grain of hinoki, and the strain distribution near the cutting edge was evaluated. The wood fracture associated with chip generation was considered as mode I fracture, and the stress intensity factor $K_{\mathrm{I}}$ for fracture mode I was calculated from the strain distribution according to the theory of linear elastic fracture mechanics for the anisotropic material. The calculated $K_{\mathrm{I}}$ increased prior to crack propagation and decreased just after the crack propagation. The change in $K_{\mathrm{I}}$ before and after crack propagation, $\Delta K_{\mathrm{I}}$, decreased in accordance with the crack propagation length, although the variance in $\Delta K_{\mathrm{I}}$ should depend on the relationships between the resolution of DIC method and the dimensions of cellular structure. The calculated $K_{\mathrm{I}}$ in this study was almost on the same order as reported in the literatures. It was also revealed, for the case of chip generation Type 0 or I, the stress intensity factor for fracture mode II could be negligible due to the higher longitudinal elastic properties of wood in the tool feed direction than the one radial ones, and the mode I fracture was dominant.
\end{abstract}

Keywords Orthogonal cutting $\cdot$ Crack propagation $\cdot$ Stress intensity factor

\section{Introduction}

In the cutting process of wood or wood-based materials, it is important to understand the mechanism of cutting, to realize better surface quality with higher efficiency. The process of wood cutting can be considered as successive controlled phenomena of wood fracture because a machined surface is produced by micro cracks continuously propagating in front of the cutting edge along with the cutting tool moving forward. Controlling these cracks is an important element for the improvement of the machined surface quality.

A part of this article was presented at the 67th Annual Meeting of the Japan Wood Research Society, Fukuoka, Japan, March 2017, and also at the 35th Annual Meeting of Wood Technological Association of Japan, Kobe, Japan, September, 2017.

Masumi Minagawa

mina@h3news1.kais.kyoto-u.ac.jp

1 Graduate School of Agriculture, Kyoto University, Kitashirakawa Oiwake-cho, Sakyo, Kyoto 606-8502, Japan

2 Daiken Corporation, 2-5-8, Kaigandori, Minami-ku, Okayama 702-8045, Japan

3 Forestry and Forest Products Research Institute, 1 Matsunosato, Tsukuba 305-8687, Japan
The study on the mechanism of wood cutting has been started since 1950s [1-3]. The studies of the dawn stage including the ones by the following researchers have been revised by recent researchers [4-11]. However, the most of the previous studies, the wood cutting was investigated mainly from the viewpoints of materials mechanics, sometimes using experimental approaches or numerical analysis. The stress and strain distribution near the cutting edge, the chip formation and the influence of cutting condition on them were also important theme. But few of the previous works discussed the cutting mechanism from the viewpoints of fracture mechanics [12-15].

On the other hand, the theory and method of the fracture mechanics have been applied to understand fracture of inhomogeneous materials $[16,17]$ and also to wood, and its relationship to the strength, anatomical features and quality of wood. The outline of the series of the works can be seen in the recent reviews [18, 19]. Many of the previous works have concentrated on the values of stress intensity factor $K_{\mathrm{I}}$ or fracture toughness $K_{\mathrm{IC}}$ of wood for the mode I fracture [20-29]. Some of them have also discussed about the mode II fracture [30-36]. The method of digital image correlation (DIC), which is a method to measure the strain of a material's surface from 
digital images, has been also applied to understand the wood fracture [37-41].

To understand the crack propagation in wood cutting with the help of fracture mechanics, we have to pay attentions to the following points; the crack propagates only in the vicinity of the cutting edge, as the result it would be influenced by the cellular structure and the depth of cut (chip thickness), and the crack may propagate according to the fracture type mode II. The influence of mode II might not be negligible, because the chip above the crack tip is compressed by the travelling cutting edge.

In this study, we conducted wood cutting experiments of small depths of cut, and the stress intensity factor $K_{\mathrm{I}}$, a parameter of linear elastic fracture mechanics (LEFM) and the fracture toughness $K_{\mathrm{IC}}$ and their variance were calculated using the DIC method referring authors previous work [42]. Moreover, we investigated the relationship of $K_{\mathrm{I}}$ values to the depth of the crack surface, and the influence of the fracture type mode II. Finally, we discussed the applicability of LEFM to the analysis of wood cutting.

\section{The application of linear elastic fracture mechanics to wood cutting}

In cutting parallel to the grain of wood, it is known that the chip types are likely to be Type 0 or I at approximately $50^{\circ}$ or less in cutting angle and $0.3 \mathrm{~mm}$ or less in depth of cut $[1-3,15]$. In the case of Type 0 , the chip is made steadily with minimal deformation and the crack length is always small. In contrast, in the case of Type I, the chip is bent as a cantilever beam after the major propagation of the crack, and the chip bends and fails around the crack tip from the increase of bending moment. In both chip types of Type 0 and I, a crack is forced to propagate by the feed of cutting tool to push the chip up. This indicates that the fracture type in both cases is the opening mode.

In LEFM, fractures are divided into three modes, I-III, from the patterns of loading to the crack, where mode I corresponds to the opening mode. When external force is applied to and around a crack tip, a stress field occurs in that area. In LEFM, the stress intensity factor is used as a parameter representing the peculiarity of the stress field, and the parameter identifies the distribution of stress, strain and displacement around the crack tip.

From the above, it is likely that applying LEFM to cutting in Type 0 and $\mathrm{I}$, which are failures in opening mode (mode I), and calculating $K_{\mathrm{I}}$, which is the stress intensity factor in mode I, may enable us to discuss the accumulation and the release of stress, and moreover, the strain energy related to crack propagation.

\section{Calculation of stress intensity factor $K_{1}$ using digital image correlation}

Assuming three axes normal to the three orthogonal planes, $x$-, $y$ - and $z$-axes, on an orthotropic material, the relationships between stresses and strains are given by the following equation:

$\left.\begin{array}{l}\varepsilon_{x}=b_{11} \sigma_{x}+b_{12} \sigma_{y} \\ \varepsilon_{y}=b_{12} \sigma_{x}+b_{22} \sigma_{y} \\ \gamma_{x y}=b_{66} \tau_{x y},\end{array}\right\}$

in the case of $x y$ plane strain, where $\sigma_{x}$ and $\sigma_{y}$ are the stresses in directions $x$ and $y, \varepsilon_{x}$ and $\varepsilon_{y}$ are the strains in directions $x$ and $y$, and $\gamma_{x y}$ and $\tau_{x y}$ are the shear strain and shear stress, respectively. In plane strain, the strain in the $z$-axis equals zero. Moreover,

$\left.\begin{array}{l}b_{11}=1 / E_{x} \\ b_{12}=-\left(\mu_{x y} / E_{x}\right)=-\left(\mu_{y x} / E_{y}\right) \\ b_{22}=1 / E_{y} \\ b_{66}=1 / G_{x y},\end{array}\right\}$

where $E_{x}$ and $E_{y}$ are the Young's moduli in directions $x$ and $y, G_{x y}$ is the shear modulus and $\mu_{x y}\left(\mu_{y x}\right)$ is the Poisson's ratio.

Concerning fracture in the opening mode (mode I) of an orthotropic material with a crack in the $x$ direction, the stresses in directions $x$ and $y, \sigma_{x}$ and $\sigma_{y}$, can be written as in the following equation $[16,17]$ :

$$
\begin{aligned}
& \sigma_{x}=\frac{K_{\mathrm{I}}}{(2 \pi r)^{1 / 2}} \frac{\beta_{1} \beta_{2}}{\beta_{2}-\beta_{1}}\left\{\frac{\beta_{2} \cos \left(\gamma_{2} / 2\right)}{\rho_{2}^{1 / 2}}-\frac{\beta_{1} \cos \left(\gamma_{1} / 2\right)}{\rho_{1}^{1 / 2}}\right\} \\
& \sigma_{y}=\frac{K_{\mathrm{I}}}{(2 \pi r)^{1 / 2}} \frac{1}{\beta_{2}-\beta_{1}}\left\{\frac{\beta_{2} \cos \left(\gamma_{1} / 2\right)}{\rho_{1}^{1 / 2}}-\frac{\beta_{1} \cos \left(\gamma_{2} / 2\right)}{\rho_{2}^{1 / 2}}\right\},
\end{aligned}
$$

where

$\left.\begin{array}{rl}\rho_{j} & =\left(\cos ^{2} \theta+\beta_{j} \sin ^{2} \theta\right)^{1 / 2} \\ \tan \gamma_{j} & =\beta_{j} \tan \theta \quad(j=1,2),\end{array}\right\}$

with $r$ and $\theta$ indicating polar coordinates at any points around the crack tip, and the origin of which is on the crack tip. In addition, $\beta_{1}$ and $\beta_{2}$ are the imaginary parts of the roots of the following equation: 
$S^{4}+\left[\left(2 b_{12}+b_{66}\right) / b_{22}\right] S^{2}+\left(b_{11} / b_{22}\right)=0$.

Substituting Eq. (3) into the equation about $\varepsilon_{y}$ in Eq. (1) gives the following equation: as the surface for observation, and resin soot was applied as a random speckle pattern after the surface was finished with a hand planer with a knife of carbon tool steel to improve the accuracy of DIC. A cutting tool was fixed to

$\varepsilon_{y}=\frac{K_{\mathrm{I}}}{(2 \pi r)^{1 / 2}} \frac{1}{\beta_{2}-\beta_{1}}\left\{\frac{\left(b_{22}-b_{12} \beta_{1}{ }^{2}\right) \beta_{2} \cos \left(\gamma_{1} / 2\right)}{\rho_{1}{ }^{1 / 2}}+\frac{\left(b_{12} \beta_{2}{ }^{2}-b_{22}\right) \beta_{1} \cos \left(\gamma_{2} / 2\right)}{\rho_{2}{ }^{1 / 2}}\right\}$.

To calculate the stress intensity factor, a least-square estimation is applied using Eq. (6), which is the theoretical strain, and $\varepsilon_{y}\left(r_{i}, \theta_{i}\right)$, which is an actual measurement of strain calculated by DIC at the coordinates $\left(r_{i}, \theta_{i}\right)$ [37, 38]. If the strains on $m$ points are gained by DIC, the error between theoretical and actual strains on each point can be calculated, and the total error $E R_{\mathrm{T}}$ is written as:

$E R_{\mathrm{T}}=\sum_{i=1}^{m}\left[\frac{K_{I}}{\left(2 \pi r_{i}\right)^{1 / 2}} \frac{A_{i}}{\beta_{2}-\beta_{1}}-\varepsilon_{y}\left(r_{i}, \theta_{i}\right)\right]^{2}$,

where,

$$
\begin{aligned}
A_{i}= & \frac{\left(b_{22}-b_{12} \beta_{1}{ }^{2}\right) \beta_{2} \cos \left(\gamma_{1 i} / 2\right)}{\rho_{1 i}{ }^{1 / 2}} \\
& +\frac{\left(b_{12} \beta_{2}{ }^{2}-b_{22}\right) \beta_{1} \cos \left(\gamma_{2 i} / 2\right)}{\rho_{2 i}{ }^{1 / 2}} \quad(1 \leq i \leq m) .
\end{aligned}
$$

As $E R_{\mathrm{T}}$ is a minimum when the differentiation of the right side of Eq. (7) with $K_{\mathrm{I}}$ equals zero, the value of $K_{\mathrm{I}}$ at that point can be considered as a likely value of $K_{\mathrm{I}}$. Therefore, Eq. (7) is differentiated with $K_{\mathrm{I}}$ and it equals zero as follows:

$$
\begin{aligned}
\frac{\partial E_{\mathrm{T}}}{\partial K_{\mathrm{I}}}= & \frac{K_{\mathrm{I}}}{\pi\left(\beta_{2}-\beta_{1}\right)^{2}} \sum_{i=1}^{m}\left[\frac{A_{i}^{2}}{r_{i}}\right] \\
& -\frac{2^{1 / 2}}{\pi^{1 / 2}\left(\beta_{2}-\beta_{1}\right)} \sum_{i=1}^{m}\left[\frac{A_{i}}{r_{i}^{1 / 2}} \varepsilon_{y}\left(r_{i}, \theta_{i}\right)\right]=0 .
\end{aligned}
$$

Solving Eq. (9) gives $K_{\mathrm{I}}$ as the following equation:

$$
K_{\mathrm{I}}=(2 \pi)^{1 / 2}\left(\beta_{2}-\beta_{1}\right) \frac{\sum_{i=1}^{m}\left[\frac{A_{i}}{r_{i}^{1 / 2}} \varepsilon_{y}\left(r_{i}, \theta_{i}\right)\right]}{\sum_{i=1}^{m}\left[\frac{A_{i}^{2}}{r_{i}}\right]} .
$$

\section{Materials and methods}

Two pieces of air-dried hinoki (Chamaecyparis obtusa), approximately $12(\mathrm{~L}) \times 5(\mathrm{R}) \times 20(\mathrm{~T}) \mathrm{mm}$ in initial size, was used as a workpiece. The density of the specimen was $0.39 \mathrm{~g} / \mathrm{cm}^{3}$. The LT surface of the workpiece was set a three-axis stage, and the workpiece was mounted on the path of the cutting tool (Fig. 1). The wedge angle of the cutting tool was $25^{\circ}$ and the clearance angle was $5^{\circ}$. The tool was made of high-speed steel (SKH51), and its rake face was coated with chromium nitride at a thickness of about $5 \mu \mathrm{m}$. Eight depths of cut were employed, that is, $40 \mu \mathrm{m}, 50 \mu \mathrm{m}, 52 \mu \mathrm{m}, 80 \mu \mathrm{m}, 105 \mu \mathrm{m}, 113 \mu \mathrm{m}, 146 \mu \mathrm{m}$ and $159 \mu \mathrm{m}$, respectively. Orthogonal cutting parallel to the grain in the RL surface was conducted.

A digital microscope (VW-6000, KEYENCE) was used to take images of the cracks that propagated in the process of cutting and their surroundings (Fig. 1). The microscope was fixed with its optical axis perpendicular to the LT surface of the workpiece. The image size employed was $1920 \times 1440$ pixels and the field of view was $0.61 \times 0.46 \mathrm{~mm}$. The shutter speed in image capturing was $1 / 125$ or $1 / 250 \mathrm{~s}$. In addition, the workpiece was lit by LED lights (OPS-S20W, Optex FA) from both right and left sides. In the cutting experiments, an image was captured before the cutting tool started to cut the workpiece, and then the workpiece was cut by feeding the cutting tool manually. The cutting procedure was temporarily suspended when the cutting edge reached the field of view. After the suspension, an image of the cutting process was captured after every tool feed step of approximate $10 \mu \mathrm{m}$, and around 50 images were taken in total. The cutting speed in this study was $18 \mu \mathrm{m} / \mathrm{min}$. It was estimated as the average of the time intervals for the tool feed of $10 \mu \mathrm{m}$. This series of operations was conducted for each depth of cut.

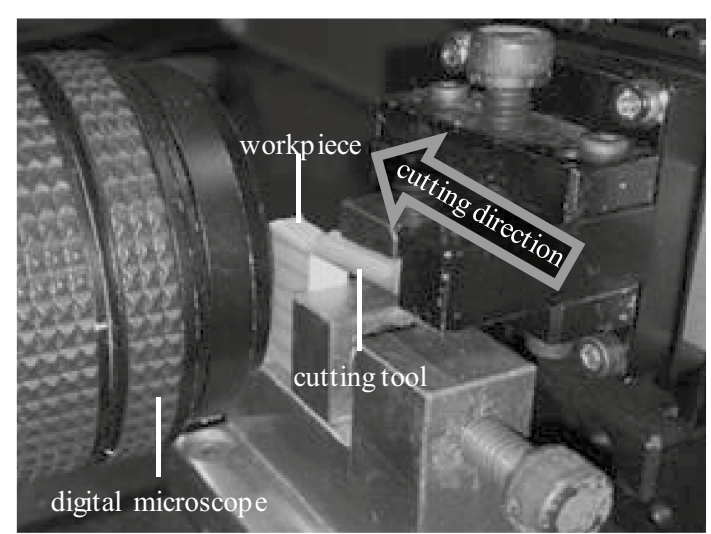

Fig. 1 Experimental apparatus in cutting experiments 
Among the images gained from the cutting experiments, the ones suitable for analysis were selected and analyzed with the DIC software coded by us in MATLAB (R2016b) language [42]. Images taken during the cutting process and an image taken before the cutting were designated as reference images and a target image, respectively. A region of interest (ROI) was defined in each reference image, and the dimension of each ROI was $320 \times 200$ pixels $(102 \times 64 \mu \mathrm{m})$ in horizontal and vertical ( $\mathrm{L}$ and $\mathrm{T}$ ) direction. The ROI position on each reference image was adjusted such that the middle point of the ROI's right edge was allocated at the crack tip (Fig. 2). The ROIs were divided into virtual grids with grid points (reference pixels) placed every ten pixels in both horizontal and vertical directions inside the ROI. In the DIC process, the displacement of all grid points between the reference and target images was estimated to calculate the strain distribution inside the ROI. The size of subset, which is the region around the reference pixels and referred to when the displacement of grid points is estimated, was set as $71 \times 71$ pixels. Although it is normal to set one ROI on the image before deformation and to compare the image with the deformed images in typical DIC analysis, in this study ROIs were set on the images during the cutting process and they were compared with the image before cutting. The locations of the crack tips were determined by manually confirming their locations on the digital images, and crack propagation lengths $(\Delta a)$ per $10 \mu \mathrm{m}$ feeding of cutting tool were calculated by comparing the locations of the crack tip between consecutive images.

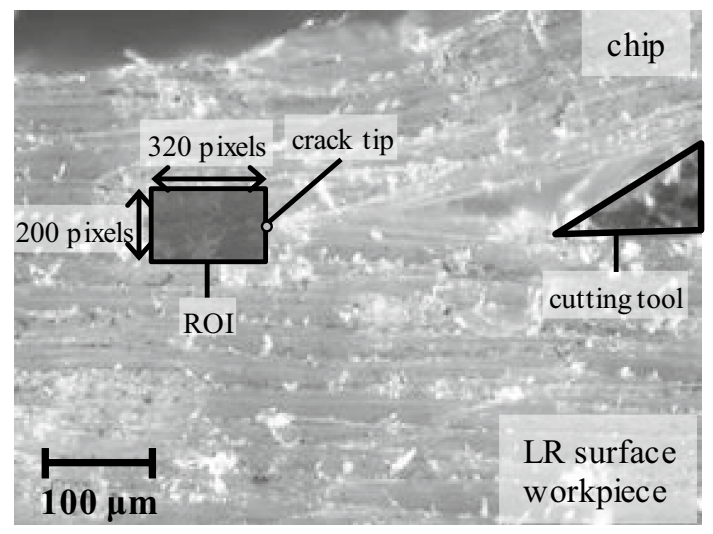

Fig. 2 An example of cutting image and region of interest (ROI)
The $x$ and $y$ directions defined in the previous section correspond to longitudinal (L) and tangential (T) directions, respectively, and the $x$ direction also corresponds to the cutting direction. Parameters $r$ and $\theta$, the polar coordinates originating at the crack tip, were calculated from the locations of the grid points after DIC analysis, and were substituted into Eq. (10) with the strains in $y$ direction calculated by DIC. The physical properties of hinoki were assumed to be as follows: $E_{\mathrm{L}}=13.1 \mathrm{GPa}, E_{\mathrm{T}}=0.62 \mathrm{GPa}, G_{\mathrm{LT}}=0.63 \mathrm{GPa}$ and $\mu_{\mathrm{LT}}=0.5$ [43].

\section{Results and discussion}

\section{Crack propagation in wood cutting}

Chip types found at each depth of cut are shown in Table 1 . The depth of the crack surface from the top surface of the workpiece (Fig. 3) for each depth of cut is also shown in Table 1 because there were some cases in which the crack did not propagate at the same height as the cutting edge

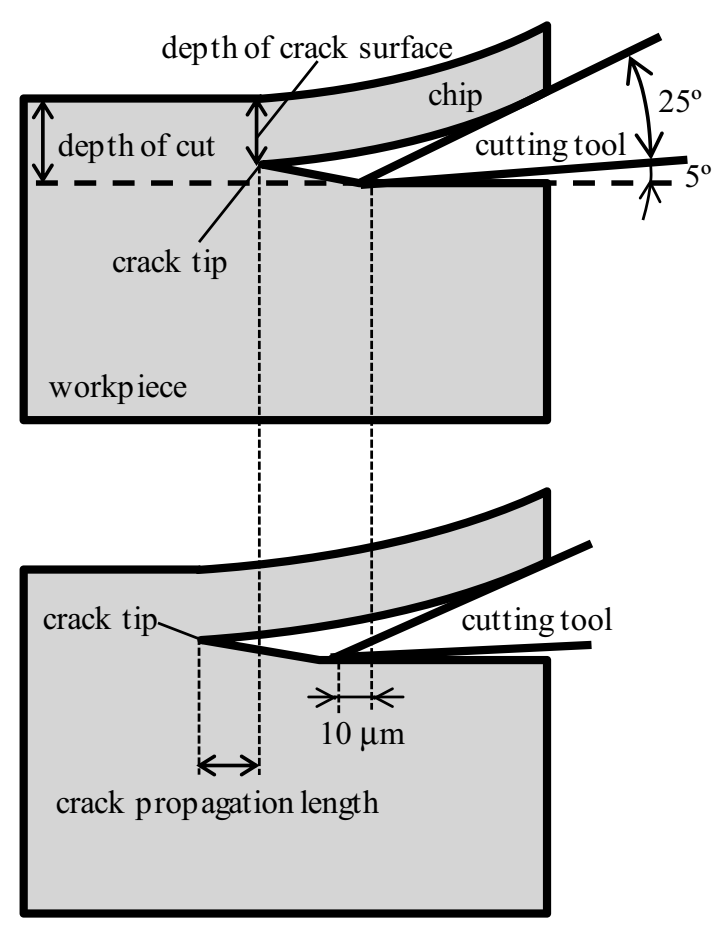

Fig. 3 Schematic diagram of depth of cut, depth of the crack surface, and crack propagation length
Table 1 Shutter speeds, observed chip types and depths of crack surface

\begin{tabular}{lllllllll}
\hline Depth of cut $(\mu \mathrm{m})$ & 40 & 50 & 52 & 80 & 105 & 113 & 146 & 159 \\
\hline Shutter speed $(\mathrm{s})$ & $1 / 250$ & $1 / 125$ & $1 / 125$ & $1 / 125$ & $1 / 250$ & $1 / 250$ & $1 / 250$ & $1 / 250$ \\
Chip type & 0 & 0 & 0 & 0 & $\mathrm{I}$ & $0 / \mathrm{I}$ & 0 & $\mathrm{I}$ \\
Depth of crack surface $(\mu \mathrm{m})$ & 40 & 61 & 52 & 80 & 88 & 86 & 139 & 111 \\
\hline
\end{tabular}


Fig. 4 Examples of two types of chip formation, Type 0 and I
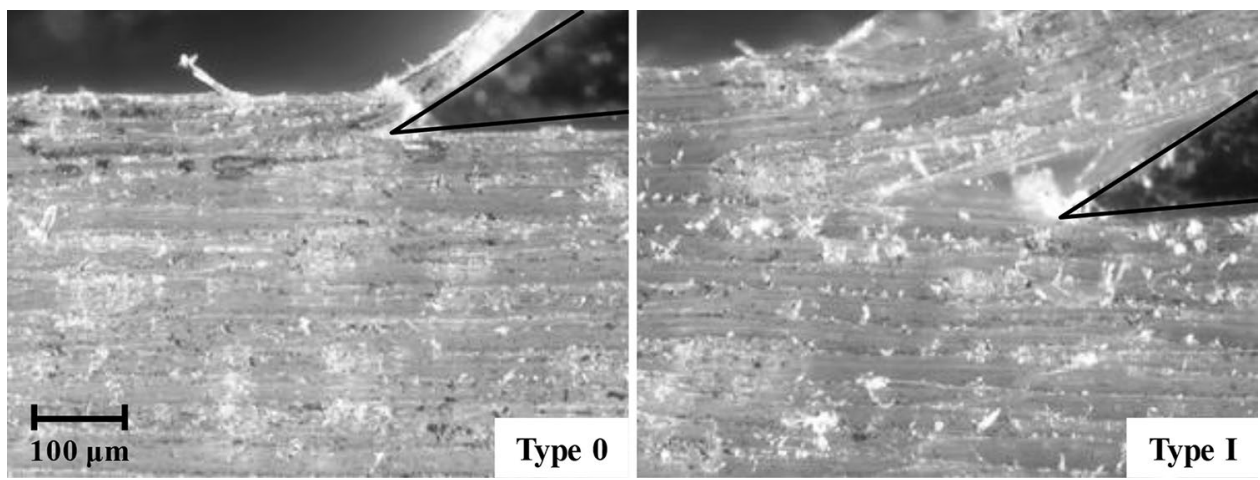

travelled. In Fig. 3, crack propagation denotes the length of the crack generated in each tool feed of $10 \mu \mathrm{m}$. Figure 4 shows the example of the images of chip Type 0 and I. Crack propagation ahead of the cutting edge as crack tip. The process of the crack generation generated by the tool feed can be considered as being fracture mode I. In $100 \mu \mathrm{m}$ or less of the depths of cut, the chip types observed were Type 0 . Type I appeared in some cases in more than $100 \mu \mathrm{m}$ of depths of cut. The chip at $113 \mu \mathrm{m}$ of depth of cut seemed to have features of both Types 0 and I.

\section{Stress intensity factor $K_{\mathrm{I}}$ near crack tip}

The stress intensity factors $K_{\mathrm{I}}$ at each travelled length of the cutting tool for two depths of cut, $40 \mu \mathrm{m}$ and $113 \mu \mathrm{m}$ are shown (black plots) in Fig. 5 a, b, respectively. At the same time, the crack propagation lengths between two consecutive images $(\Delta a)$ at each travelled length of the cutting tool are shown as bars. The values of $\Delta a$ are the crack propagation length between two moments at which $K_{\mathrm{I}}$ were calculated, so that the bars of $\Delta a$ are allocated between plots of $K_{\mathrm{I}}$. Hereby, the travelled length of the cutting tool denotes the distance the cutting tool was fed from the moment at which an image was acquired just after the start of tool feed of $10 \mu \mathrm{m}$. In this analysis, the moment of crack propagation is so defined as the time of event when the apparent crack propagation was confirmed visually by compering a couple of serious digital images. It was confirmed that there was a tendency for $K_{\mathrm{I}}$ to increase when the crack propagation lengths were small, and to decrease when the crack propagation lengths were large. The value of $K_{\mathrm{I}}$ periodically fluctuated as the cutting tool travelled.

To clarify the relationship between the crack propagation length and $K_{\mathrm{I}}$, the difference of two consecutive $K_{\mathrm{I}}$ values, $\Delta K_{\mathrm{I}},\left(\Delta K_{\mathrm{I}}=\Delta K_{\mathrm{I} n+1}-\Delta K_{\mathrm{I} n}, n=1,2 \ldots\right)$ was calculated, and its relationship to the square root of the crack propagation length $\left(\Delta a^{1 / 2}\right)$ was examined. The result is shown in Fig. 6 . All the results for different depths of cut are plotted in the figure altogether. A negative correlation was found between (a) depth of cut: $40 \mu \mathrm{m}$

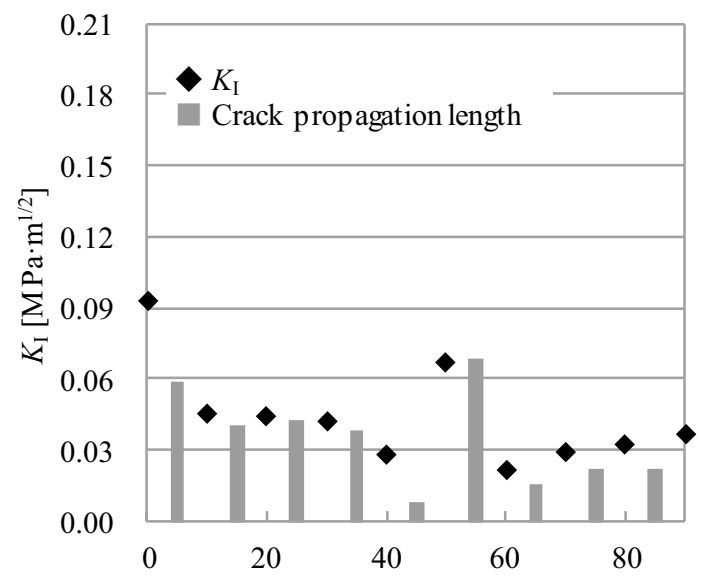

(b) depth of cut: $113 \mu \mathrm{m}$

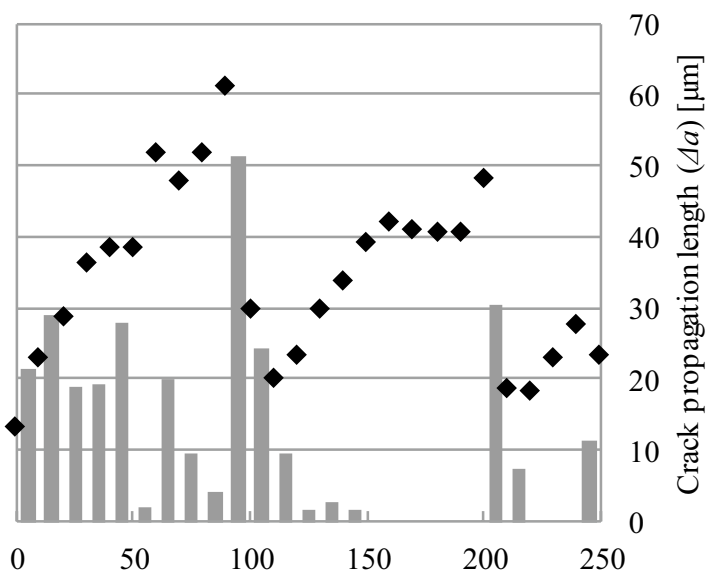

Travelled length of cutting tool $[\mu \mathrm{m}]$

Fig. 5 Changes of stress intensity factor $K_{\mathrm{I}}$ and the crack propagation lengths $(\Delta a)$. a $40 \mu \mathrm{m}$ and $\mathbf{b} 113 \mu \mathrm{m}$ of depths of cut. A bar indicates the crack propagation length between two consecutive images and a black plot indicates $K_{\mathrm{I}}$ at each travelled length of the cutting tool 


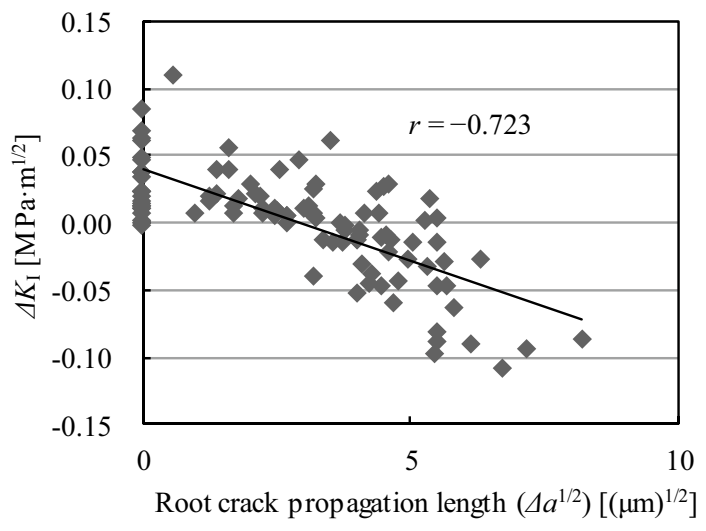

Fig. 6 Relationship between the square root of crack propagation length $\left(\Delta a^{1 / 2}\right)$ and $\Delta K_{\mathrm{I}}$

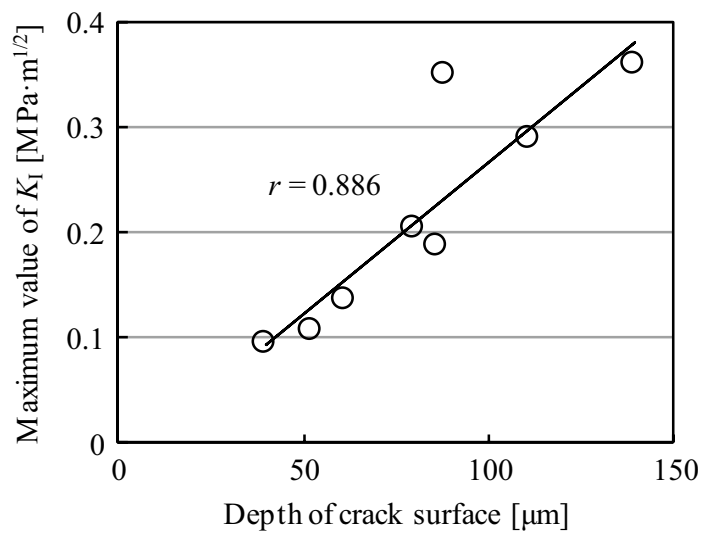

Fig. 7 Relationship between depth of the crack surface and maximum value of $K_{\mathrm{I}}$ in each depth of cut

$\Delta a^{1 / 2}$ and $\Delta K_{\mathrm{I}}$, although relatively large disperse in $\Delta K_{\mathrm{I}}$ exists.

The results of Figs. 5 and 6 indicate that the larger propagation of crack causes more decrease in $K_{\mathrm{I}}$, and thus larger amount of strain energy released at the crack propagation. This relationship between crack propagation and the change in $K_{\mathrm{I}}$ is consistent with the general idea of fracture mechanics, where the amount of crack propagation is explained to increase in accordance with the energy release per crack increase according to the Griffith's theory [44]. However, the plots in Fig. 6 dispersed. This can be attributed to anatomical features of wood specimen; layer structure consisted of tracheid walls and ray tissues and their variance. Due to the heterogeneity of the workpiece, the initiation of the crack propagation, crack length and direction varied largely, and the resultant amount of strain energy released at each crack propagation also varied.

\section{Influence of depth of crack on $K_{\mathrm{I}}$}

The relationship between the maximum value of $K_{\mathrm{I}}$ for each depth of cut and the depth of the crack surface was considered (Fig. 7). There was a positive correlation between the depth of the crack surface and the maximum value of $K_{\mathrm{I}}$ in Fig. 7. Assuming the maximum value of $K_{\mathrm{I}}$ is the fracture toughness, which is the limit $K_{\mathrm{I}}$ could take, the result in Fig. 7 indicates that the fracture toughness varies with the depth of the crack surface.

It is generally accepted that each material has a particular fracture toughness value, but under conditions where one side of the crack is extremely thin, like the cutting experiments in this study, it showed fracture toughness changes depending on how thin that side is. One possible reason for this tendency is the stress concentration in thinner side. As the depth of the crack surface becomes smaller, a greater concentration of stress occurs around the crack tip and crack propagates with less strain energy. This suggests that the stress concentration decreases inversely with the depth of the crack surface, and the fracture toughness will average at around the specific value of the material.

The maximum $K_{\mathrm{I}}$ value for each depth of cut ranged from 0.09 to $0.36 \mathrm{MPa} \cdot \mathrm{m}^{1 / 2}$ in this study. The fracture toughness, $K_{\text {IC }}$, is the stress intensity factor at which a thin crack in the material begins to grow, and it is a measure of a material's resistance to brittle fracture. The values of fracture toughness $K_{\mathrm{IC}}$ of wood reported in the former studies take the values from 0.2 to $1.0 \mathrm{MPa} \cdot \mathrm{m}^{1 / 2}[14,21]$. The maximum $K_{\mathrm{I}}$ values in this study were slightly smaller than the generally accepted $K_{\text {IC }}$ values of wood [20-29]. The reason for the discrepancy is not clear. However, one possible reason could be that the strain energy which accumulated before crack propagation was smaller due to the less wood substance in the thinner chip. As the result, $K_{\mathrm{IC}}$ was estimated lower. The maximum values of $K_{\mathrm{I}}$ increased with the depth of the crack surface in Fig. 7 supports this reasoning. However, the values estimated in this study are almost in the same order of those in the literatures.

Calculation of $K_{\mathrm{I}}$ using strains in $102(\mathrm{~L}) \times 64(\mathrm{R}) \mu \mathrm{m}$ region could be also a factor of $K_{\mathrm{I}}$ being small. It is possible that some local variance in physical properties due to cell walls and intercellular layers could have lowered $K_{\mathrm{I}}$ value. The validity of applying physical properties obtained by experiments in the literatures using specimen much larger than those in this study to the calculation of $K_{\mathrm{I}}$ should be discussed more in detail.

\section{Applicability of LEFM to wood cutting}

In this study, crack propagation in the cellular structure was analyzed in a scale much smaller than those normally discussed in the literatures. According to the theory of LEFM, 
there is no scale limit for its application, so long as the anisotropy of the material properties is properly taken into account. But we have to pay attention the variance in material properties due to the anatomical features on the variation of the calculated $K_{\mathrm{I}}$.

We have got four evidences for the applicability of LEFM to wood cutting; from the image of cutting process in Fig. 4, the crack propagation ahead of the cutting edge can be categorized as fracture mode I. The calculated $K_{\mathrm{I}}$ using the data obtained by DIC method (Fig. 5), increased prior to crack propagation and decreased just after the crack propagation. The values of $\Delta K_{\mathrm{I}}$ decreased in accordance with the crack propagation length (Fig. 6), although the variance in $\Delta K_{\mathrm{I}}$ could depend on the relationships between the resolution of DIC method and the dimensions of cellular structure. The calculated $K_{\mathrm{I}}$ in this study was almost on the same order as reported in the literatures.

On the other hand, the result of Fig. 7 shows, $K_{\mathrm{I}}$ is influenced by the depth of crack. This could be attributed to the deviation of the crack position in the specimen due to the removal of thin chip from the wood surface. This situation is not supposed in LEFM.

From the discussion above, it can be concluded that the mechanism of chip generation in longitudinal orthogonal wood cutting can be discussed using the theory of LEFM, although we have to pay attention to the influence of the variance in physical or mechanical properties of wood in the region of interest on the variance of $K_{\mathrm{I}}$.

\section{Analysis on mixture of fracture modes I and II}

Fracture mode II of wood and the stress intensity factor for this mode were also discussed by a few researchers [30-36]. Yoshihara intensively discussed the mode II fracture not only for solid wood but also for medium density fiberboard $[34,36]$. In the process of wood cutting in this study, the generated chip is pressed by the travelling cutting edge in feed direction, that is, in the longitudinal direction of wood, so that we have evaluated the stress intensity factors $K_{\mathrm{I}}$ and $K_{\mathrm{II}}$ for the fracture modes I and II, respectively. The factors were calculated in the same manner as in the previous sections, but with the following formula as one of the basic formula:

$\varepsilon_{y}(r, \theta)=\frac{K_{\mathrm{I}}}{(2 \pi r)^{1 / 2}} \frac{f(\theta)}{\beta_{2}-\beta_{1}}+\frac{K_{\mathrm{II}}}{(2 \pi r)^{1 / 2}} \frac{g(\theta)}{\beta_{2}-\beta_{1}}$.

Hereby $f(\theta)$ and $g(\theta)$ are the functions of $\theta$ for two fracture modes I and II, respectively. They are introduced by the same way as for formulas (4) and (6).

Figure 8 shows changes in the stress intensity factors $K_{\mathrm{I}}$ and $K_{\mathrm{II}}$ and the crack propagation length $(\Delta a)$ for $80 \mu \mathrm{m}$ of

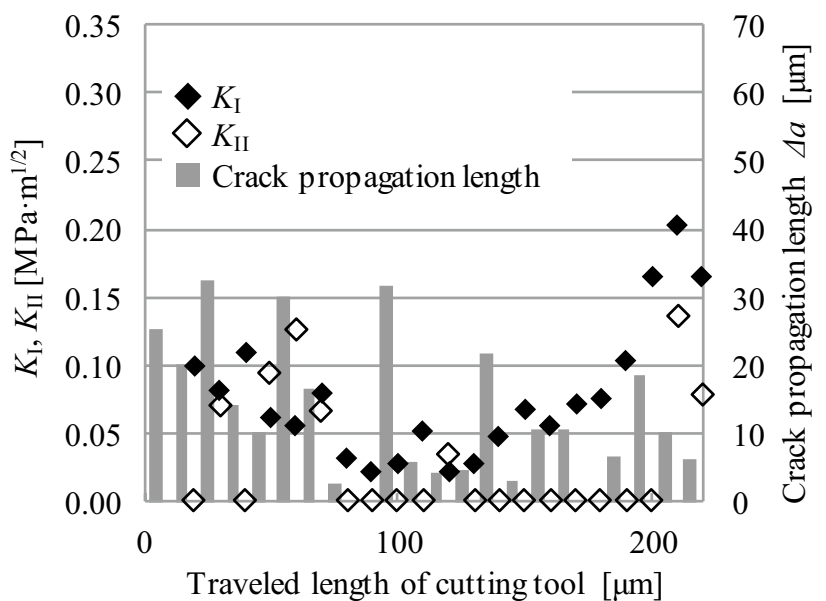

Fig. 8 Changes of stress intensity factor $K_{\mathrm{I}}, K_{\mathrm{II}}$ and crack propagation lengths $(\Delta a) .80 \mu \mathrm{m}$ of depths of cut. A bar indicates the crack propagation length between two consecutive images. A black plot and a white plot indicate $K_{\mathrm{I}}$ and $K_{\mathrm{II}}$ at each travelled length of the cutting tool, respectively

depth of cut. There were some cases, the values of $K_{\mathrm{II}}$ (white plots) took the values almost same as the ones of $K_{\mathrm{I}}$ (black plots); however, they took mostly small values, or negative small ones in some cases. This can be attributed to the fact that the stress component in the normal direction associated with $K_{\mathrm{II}}$ was very small and it was not detected significantly by DIC method, and as a result, the error in the calculated values became relatively larger. In Fig. 8, the plots for negative small values of $K_{\text {II }}$ were considered as zero. It is confirmed, Type 0 chip was generated in these cases. Moreover, as the chip generation in Fig. 4 shows, the fracture mode I was dominant in both cases of Type 0 and I chip generation in the orthogonal longitudinal cutting. This tendency was conformed for all cases of depths of cut. The compression strain in the direction of crack propagation discussed on the mode II fracture generates correspondingly in the longitudinal direction in this study, that is, in the direction of the highest elasticity, so that the compression or buckling strain associated to mode II fracture could be negligible.

\section{Conclusions}

The method of DIC was applied to the digital image of orthogonal cutting parallel to the grain of hinoki, and the strain distribution near the cutting edge was evaluated. The fracture associated with chip generation was considered as mode I fracture and the stress intensity factor $K_{\mathrm{I}}$ was calculated from the strain distribution according to the theory of 
LEFM for the anisotropic material. From the change in $K_{\mathrm{I}}$, it can be concluded that the mechanism of chip generation in the longitudinal orthogonal wood cutting can be discussed using the theory of LEFM, although we have to pay attention to the influence of the variance in elastic moduli and Poisson's ratios of wood in the region of interest on the variance of $K_{\mathrm{I}}$. In addition, for the case of chip generation Type 0 or I, the fracture of mode I was dominant in the crack propagation near the cutting edge.

Acknowledgements Authors would like to express sincere thanks to Kanefusa Corporation for providing the cutting tools.

\section{References}

1. Franz NC (1955) Analysis of chip formation in wood machining. For Prod J 5:332-336

2. Franz NC (1958) Analysis of the wood-cutting process. Dissertation, University of Michigan, USA

3. McKenzie WM (1967) The basic wood cutting process. In: Proceedings of the second wood machining seminar. Oct 10-11 1967, Richmond, pp 3-8

4. Wyeth DJ, Goli G, Atkins AG (2009) Fracture toughness, chip types and the mechanics of cutting wood. A review COST Action E35 2004-2008: Wood machining-micromechanics and fracture. Holzforschung 63:168-180. https://doi.org/10.1515/HF.2009.017

5. Orlowski KA, Ochrymiuk T, Akins A, Chuchala D (2013) Application of fracture mechanics for energetic effects predictions while wood sawing. Wood Sci Technol 47:949-963. https://doi. org/10.1007/s00226-013-0551-x

6. Orlowski KA, Ochrymiuk T, Sandak J, Sandak A (2013) Estimation of fracture toughness and shear yield stress of orthotropic materials in cutting with rotating tools. Eng Fract Mech 178:433-444

7. Orlowski KA, Ochrymiuk T (2014) Fracture mechanics model of cutting power versus widespread regression equations while wood sawing with circular saw blades. Ann WULS SGGW For Wood Technol 85:168-174

8. Nairn JA (2015) Numerical simulation of orthogonal cutting using the material point method. Eng Fract Mech 149:262-275

9. Hlásková L, Kopecký Z (2015) Fracture toughness and shear stresses determining od chemically modified beech. Ann WULS SGGW For Wood Technol 90:73-78

10. Williams JG, Patel Y (2016) Fundamentals of cutting. Interface Focus 6:20150108

11. Nairn JA (2016) Numerical modelling of orthogonal cutting: application to woodworking with a bench plane. Interface Focus 6:20150110

12. Merhar M, Bučar B (2012) Cutting force variability as a consequence of exchangeable cleavage fracture and compressive breakdown of wood tissue. Wood Sci Technol 46(5):965-977

13. Ohya S, Ohta M, Okano T (1992) Analysis of wood slicing mechanism along the grain I. Previous check occurrence conditions. Mokuzai Gakkaishi 38:1098-1104

14. Triboulot P, Asano I, Ohta M (1983) An application of fracture mechanics to the wood-cutting process. Mokuzai Gakkaishi 29:111-117

15. Matsuda Y, Fujiwara Y, Fujii Y (2015) Observation of machined surface and subsurface structure of hinoki (Chamaecyparis obtusa) produced in slow-speed orthogonal cutting using X-ray computed tomography. J Wood Sci 61:128-135
16. Wu EM (1963) Application of fracture mechanics to orthotropic plates. TAM Rept 248

17. Corten HT (1972) Fracture mechanics of composites. In: Liebowitz $\mathrm{H}$ (ed) Fracture of nonmetals and composites. Academic Press, New York, pp 675-769

18. Conrad MPC, Smith GD, Fernlund G (2003) Fracture of solid wood: a review of structure and properties at different lenght. Wood Fiber Sci 35(4):570-584

19. Merhar M, Bučar DG, Bučar B (2013) Mode I critical stress intensity factor of beech wood (Fagus Sylvatica) in a TL configuration: a comparison of different method. Drvna Ind 64(3):221-229

20. Prokopskit G (1993) The application of fracture mechanics to the testing of wood. J Mater Sci 28:5995-5999

21. Tokuda M (1998) Relationship between critical stress-intensity factor KIC and crack size induced by nailing for different typical wood species of timber construction (in Japanese). J Soc Mat Sci 47:356-360

22. Sato K, Yamamoto H, Taya A, Okuyama H (2000) Influence of moisture content on critical stress intensity factor of wood (in Japanese). J Soc Mat Sci 49:365-367

23. Yoshihara H (2007) Simple estimation of critical stress intensity factors of wood by tests with double cantilever beam and threepoint end-notched flexure. Holzforschung 61:182-189. https://doi. org/10.1515/HF.2007.032

24. Yoshihara H (2010) Examination of the Mode I critical stress intensity factor of wood obtained by single-edge-notched bending tests. Holzforschung 64:501-509

25. Yoshihara $H$ (2010) Influence of bending conditions on the measurement of mode I critical stress intensity factor for wood and medium density fiberboard by the single-edge-notched tension test. Holzforschung 64:734-735

26. Yoshihara H, Usuki A (2011) Mode I critical stress intensity factor of wood and medium-density fiber board measured by compact tension test. Holzforschung 65:729-735

27. Yoshihara H, Ataka N, Maruta M (2017) Measurement of the Mode I critical stress intensity factor of solid wood and mediumdensity fiberboard by four-pint single-edge-notched bending (4SENB) test (in Japanese). J Soc Mat Sci 66:323-327

28. Ochiai Y, Aoki K, Inayama M (2017) Fundamental research of an evaluation method of splitting failure in timber I. Differences of wood species and evaluation method of splitting failure by the CT test specimen (in Japanese). Mokuzai Gakkaishi 63:269-276

29. Pitti RM, Chazal C, Labesse-Jied F, Lapusta Y (2017) Stress intensity factors for viscoelastic axisymmetric problems applied to wood. Exp Appl Mech 4:89-96

30. Barrett JD, Foschi RO (1977) Mode II stress-intensity factors for cracked wood beams. Eng Fract Mech 9(2):371-378

31. Murphy JF (1988) Mode II wood test specimen: beam with center slit. J Test Eval 16(4):364-368

32. Yoshihara H (2008) Mode II fracture mechanics properties of wood measured by asymmetric four-point bending test using a single-edge-notched specimen. Eng Fract Mech 75:4727-4739

33. Yoshihara H, Sato A (2009) Shear and crack tip deformation correction for the double cantilever beam and three-point endnotched flexure specimen for mode I and mode II fracture toughness measurement of wood. Eng Fract Mech 76:335-346

34. Yoshihara H (2010) Mode I and mode II initiation fracture toughness and resistance curve of medium density fiberboard measured by double cantilever beam and three-point bend end-notched flexure tests. Eng Fract Mech 77:2537-2549

35. Yoshihara H (2012) Mode II critical stress intensity factor of wood measured by the asymmetric four-point bending test using single-edge-notched specimen while considering an additional crack length. Holzforschung 66:989-992

36. Yoshihara H (2013) Mode II critical stress intensity factor of medium density fiberboard measured by asymmetric four-point 
bending test analysis of kink crack formation. BioResources 8(2):1771-1789

37. McNeill SR, Peters WH, Sutton MA (1987) Estimation of stress intensity factor by digital image correlation. Eng Fract Mech 28:101-112

38. Yoneyama S, Takashi M (2001) Automatic determination of stress intensity factor utilizing digital image correlation (in Japanese). $\mathrm{J}$ JSEM 1:202-206

39. Samarasinghe S, Kulasiri D (2004) Stress intensity factor of wood from crack-tip displacement fields obtained from digital image processing. Silva Fenn 38(3):267-278

40. Zhao J, Zhao D (2013) Evaluation of mixed-mode stress intensity factor of wood from crack-tip displacement fields utilizing digital image correlation. WSEAS Trans Appl Theor Mech 8(4):282-291
41. Tukiainen P, Hughes M (2016) The cellular level mode I fracture behavior of spruce and birch in the RT crack propagation system. Holzforschung 70:157-165

42. Matsuda Y, Fujiwara Y, Murata K, Fujii Y (2017) Residual strain analysis with digital image correlation method for subsurface damage evaluation of hinoki (Chamaecyparis obtusa) finished by slow-speed orthogonal cutting. J Wood Sci 63:615-624

43. Nakato K (ed) (1985) Shinpen mokuzai kōgaku (in Japanese). Yōkendō, Tokyo, p 142

44. Smith I, Landis E, Gong M (2003) Fracture and fatigue in wood. Wiley, West Sussex, p 242 